\title{
Diversidad genética de piracanjuba usada en programas de repoblación con marcadores microsatélites
}

\author{
Maria del Pilar Rodriguez-Rodriguez(1), Nelson Mauricio Lopera-Barrero(1), Ricardo Pereira Ribeiro(1), \\ Jayme Aparecido Povh ${ }^{(2)}$, Lauro Vargas ${ }^{(1)}$, Rodolfo Nardez Sirol ${ }^{(3)}$ y Carolina Bespalhok Jacometo ${ }^{(1)}$
}

\begin{abstract}
(1)Universidade Estadual de Maringá, Departamento de Zootecnia, Núcleo de Pesquisa PeixeGen, Av. Colombo, no 5.790, Bloco J57, Sala 8B, CEP 87020-900 Maringá, PR. E-mail: pilar.peixegen@gmail.com, nelson.peixegen@gmail.com, ricardo.peixegen@gmail.com, Ivargas@uem.br, carolina.peixegen@gmail.com, (2)Universidade Federal de Mato Grosso, Instituto de Ciências Agrárias e Tecnológicas, Rodovia Rondonópolis-Guiratinga, Km 06, CEP 78735-910 Rondonópolis, MT. E-mail: jayme.peixegen@gmail.com ${ }^{(3)}$ Companhia Paulista de Força e Luz - Geração, Rodovia Campinas - Mogi Mirim, Km 2,5, CEP 13088-900 Campinas, SP. E-mail: rnsirol@cpfl.com.br
\end{abstract}

Resumen - El objetivo de este trabajo fue estimar la diversidad genética de un lote de Brycon orbignyanus usado en programas de repoblación, a través de marcadores microsatélites. Se analizaron muestras de 44 reproductores, de 70 larvas y de 69 alevinos, con la amplificación de cinco loci descritos para Brycon opalinus. El número de alelos, la heterozigosidad observada (Ho) y esperada (He), el índice de Shannon (IS), la diversidad genética de Nei (DGN), el coeficiente de endogamia $\left(\mathrm{F}_{\text {is }}\right)$, la distancia (DG) e identidad genética (IG), el número efectivo de alelos, el test del equilibrio de Hardy-Weinberg (EHW) y el desequilibrio de ligación fueron calculados. Reproductores y progenie tuvieron un número similar de alelos en los loci evaluados. La Ho media, IS, DGN, DG e IG mostraron que existe menor distancia genética entre parentales y larvas y una disminución de variabilidad genética en los alevinos. Fueron observados desvíos en EHW y desequilibrio de ligación en seis pares de loci. El $\mathrm{F}_{\text {is }}$ mostró exceso de heterocigotos en parentales y larvas y déficit de heterocigotos en los alevinos. El lote de reproductores está en proceso de pérdida de alelos y hubo disminución de la variabilidad genética entre la fase de larva y alevino.

Términos para indexación: Brycon orbignyanus, alevino, genética de la conservación, larva, manejo reproductivo, variabilidad genética.

\section{Genetic diversity of piracanjuba used in stock enhancement programs with microsatellite markers}

\begin{abstract}
The objective of this work was to estimate the genetic diversity of a Brycon orbignyanus lot used in stock enhancement programs, using microsatellite markers. Samples of 44 broodstocks, 70 larvae and 69 fingerlings, were analyzed with amplification of five loci described for Brycon opalinus. The number of alleles, the observed (Ho) and expected (He) heterozygosity, Shannon index (IS), Nei's genetic diversity (DGN), the inbreeding coefficient $\left(\mathrm{F}_{\mathrm{is}}\right)$, distance (DG) and genetic identity (IG), the effective number of alleles, the test of Hardy-Weinberg equilibrium (EHW) and the linkage disequilibrium were calculated. Broodstocks and offspring had a similar number of alleles at the tested loci. Ho average, IS, DGN, DG and IG showed that there is less genetic distance between parental and larvae and a decrease of genetic variability in the fingerlings. Deviations in the EHW and disequilibrium ligation were observed in six pairs of loci. Inbreeding coefficient showed excess of heterozygotes in parental and larvae and heterozygote deficiencies in fingerlings. The broodstock is in process of allele losses and the genetic variability decreased between larva and fingerling stages.
\end{abstract}

Index terms: Brycon orbignyanus, fingerling, conservation genetics, larvae, reproductive management, genetic variability.

\section{Introducción}

En Brasil, como en otros países, se verifica una disminución en la producción pesquera en consecuencia del declino en la captura, y un indicador biológico de esta disminución es la situación actual de las especies de peces nativas migratorias (Taylor et al., 2006). Las principales amenazas a la ictiofauna están relacionadas a las actividades humanas que alteran la calidad de los ecosistemas acuáticos, marcadamente del uso inadecuado del agua y del suelo. Agostinho et al. (2005) destacan como causas principales de la reducción de la 
biodiversidad, en ecosistemas acuáticos continentales brasileños, la contaminación y eutrofización, la construcción de hidroeléctricas y el consecuente control de crecientes, la pesca indiscriminada y la introducción de especies exóticas.

Las especies del genero Brycon, conocidas regionalmente como matrinchãs, piracanjubas, piraputangas o pirapitingas son migratorias, de desova total, que habitan ríos de aguas limpias y con alta oxigenación. Representan importantes fuentes de pesca en las regiones en que se encuentran presentes y son de gran importancia económica (Lopera Barrero, 2007). El piracanjuba, Brycon orbignyanus (Valenciennes, 1849) (Characiformes, Characidae, Bryconinae), es una especie nativa de las cuencas formadas por los ríos Uruguay y Paraná (Zaniboni Filho et al., 2006) que ha despertado un gran interés por parte de investigadores y productores por su elevado valor, precocidad, buena conversión alimentar y buena aceptación de alimentos artificiales (Borba et al., 2006). La desaparición de poblaciones de los principales locales de pesca, es preocupante, y el piracanjuba actualmente es catalogado como especie en riesgo de extinción (Machado, 2005).

De las acciones empleadas para mitigar estos impactos, los programas de repoblación son comúnmente utilizados (Lopera Barrero et al., 2007), sin embargo, esta práctica que ha sido implementada hace más de tres décadas en Brasil no cuenta con un respaldo científico (Agostinho et al., 2005). Según Povh et al. (2008a), las introducciones de peces de forma no planeada pueden provocar una reducción de la variabilidad genética, por manejos genéticos y reproductivos inadecuados, y pueden provocar erosión genética en las poblaciones naturales receptoras de alevinos liberados. Así, con el propósito de implementar un programa de conservación eficiente, se debe considerar la preservación genética de las poblaciones de peces nativos, para lo cual es importante obtener una identificación y caracterización de procedimientos genéticos y reproductivos utilizados en lotes de esta especie, cuando orientados a la repoblación (Sirol \& Britto, 2006). Un ejemplo de ello es el estudio genético de progenies que serán liberadas en los ríos, las cuales son analizadas principalmente mediante la colecta de muestras en la fase de larva (Lopera Barrero et al., 2008c, Povh et al., 2008b). Normalmente, esas progenies son liberadas a los ríos en la fase de alevino ( 60 a 90 días de vida), y es posible que la influencia de factores como el canibalismo
(Borba et al., 2006), medio ambiente (Pedreira, 2003) y mortalidad durante el crecimiento redistribuyan las características poblacionales y, consecuentemente, su variabilidad genética.

El objetivo de este trabajo fue estimar la diversidad genética de un lote de $B$. orbignyanus usado en programas de repoblación, a través de marcadores microsatélites.

\section{Materiales y Métodos}

Se utilizaron 44 reproductores (22 machos y 22 hembras) de B. orbignyanus, seleccionados de un lote mantenido en cautiverio en las instalaciones de la Estación de Acuicultura e Hidrología de la Duke Energy International, ubicada en la ciudad de Salto Grande $\left(23^{\circ} 10^{\prime} \mathrm{S}, 49^{\circ} 13^{\prime} \mathrm{W}\right)$, São Paulo, Brasil. El lote fue formado a partir de reproductores capturados en el rio Paraná, pertenecientes a dos pisciculturas localizadas en las ciudades de Castilho y Porto Ferreira en el Estado de São Paulo. Este lote permaneció inalterado, sin introducción de nuevos individuos, durante seis años, desde el momento de su formación. Los individuos son utilizados actualmente en programas de repoblación realizados en el río Paranapanema, São Paulo.

El experimento se realizó en enero de 2008. Los peces fueron inducidos a reproducción con extracto de hipófisis de carpa. Las hembras recibieron $5,5 \mathrm{mg} \mathrm{kg}^{-1}$, divididos en dos aplicaciones: $10 \%$ del total en la primera aplicación, y el $90 \%$ restante 12 horas después. Los machos recibieron $2,5 \mathrm{mg} \mathrm{kg}^{-1}$ en dosis única, paralelamente con la ultima dosis de las hembras. La reproducción simultánea de los 44 reproductores se realizó por el sistema reproductivo seminatural descrito por Lopera Barrero (2007).

Después de seis horas de colecta de huevos, fue verificado por presión abdominal si los machos y hembras utilizados desovaron completamente: la falta de salida de gametos indicaba que ocurrió liberación total de ellos. De todos los individuos se colectaron muestras de aleta caudal, de $0,5 \mathrm{~cm}^{2}$ aproximadamente, $\mathrm{y}$ se almacenaron en microtubos de $1,5 \mathrm{~mL}$ con alcohol etílico absoluto, para la posterior extracción y amplificación del ADN. El porcentaje de mortalidad de los reproductores usados en los cruzamientos se definió un día después del proceso reproductivo.

Tres días después de la eclosión de los huevos, 200 larvas aproximadamente se recolectaron de forma 
aleatoria de todas las incubadoras y se colocaron en microtubos de $1,5 \mathrm{~mL}$ con alcohol etílico absoluto. De esas larvas, 70 se escogieron aleatoriamente para posterior extracción del ADN. Noventa días después, se recolectaron muestras de aleta caudal de 200 alevinos, pertenecientes a la misma desova. De esos alevinos, 69 se escogieron para la posterior extracción de ADN.

Para la extracción de ADN, se utilizó la metodología descrita por Lopera Barrero et al. (2008a). En microtubos con las aletas, se adicionaron $550 \mu \mathrm{L}$ de buffer de lisis $\left(50 \mathrm{mmol} \mathrm{L}^{-1}\right.$ Tris- $\mathrm{HCl}, 50 \mathrm{mmol} \mathrm{L}^{-1}$ EDTA, $100 \mathrm{mmol} \mathrm{L}^{-1} \mathrm{NaCl}, 1 \% \mathrm{SDS}$ ) y $200 \mu \mathrm{g} \mathrm{mL}^{-1}$ de proteinasa $\mathrm{K}$. Las muestras se incubaron en baño maría a $50^{\circ} \mathrm{C}$ por 12 horas. El ADN se precipitó con $600 \mu \mathrm{L}$ de solución de $\mathrm{NaCl}\left(5 \mathrm{~mol} \mathrm{~L}^{-1}\right)$ y se centrifugó por $10 \mathrm{~min}$ a $12.000 \mathrm{rpm}$. El ADN se transfirió para nuevos micro-tubos, se precipitó con $700 \mu \mathrm{L}$ de alcohol etílico absoluto frío y se incubó por dos horas a $-20^{\circ} \mathrm{C}$; enseguida, se lavó con $700 \mu \mathrm{L}$ de alcohol etílico $70 \%$, se suspendió en $80 \mu \mathrm{L}$ de solución amortiguadora TE $\left(10 \mathrm{mmol} \mathrm{L}^{-1}\right.$ Tris $\mathrm{pH} 8,1 \mathrm{mmol} \mathrm{L}^{-1}$ EDTA) y se mezcló con $30 \mu \mathrm{g} \mathrm{mL}^{-1}$ de RNAasa. Las muestras se incubaron por $40 \mathrm{~min}$ en baño maría a $37^{\circ} \mathrm{C}$ y se conservaron a $-20^{\circ} \mathrm{C}$.

ElADN se cuantificó en espectrofotometro Shimadzu UV 1601, con amplitud de onda de $260 \mathrm{~nm}$, y se diluyó para una concentración de 10 e $5 \mathrm{ng} \mu \mathrm{L}^{-1}$, para aletas y larvas, respectivamente. La integridad del ADN se verificó en electroforesis horizontal en gel de agarosa $1 \%$, a $70 \mathrm{~V}$ por $60 \mathrm{~min}$, en solución amortiguadora TBE $1 \mathrm{X}$ (500 mmol L-1 Tris-HC1, $60 \mathrm{mmol} \mathrm{L}^{-1}$ ácido bórico, $83 \mathrm{mmol} \mathrm{L}^{-1}$ EDTA). El gel se marcó con bromuro de etidio $\left(0,5 \mu \mathrm{g} \mathrm{mL}^{-1}\right)$ por $30 \mathrm{~min}$, y la imagen se capturó con el sistema fotográfico EDAS (Kodak 1D Image Analysis 3.5).

El ADN se amplificó en un volumen final de reacción de $18 \mu \mathrm{L}$. Se utilizó buffer Tris-KCl, $2 \mathrm{mmol} \mathrm{L}^{-1} \mathrm{MgCl}_{2}$, $0,8 \mu \mathrm{mol} \mathrm{L}^{-1}$ de cada iniciador (forward y reverse), $0,2 \mathrm{mmol} \mathrm{L}^{-1}$ de cada dNTP, una unidad de Platinum Taq ADN polimerasa, 10 y $20 \mathrm{ng} \mu \mathrm{L}^{-1}$ de $\mathrm{ADN}$, para larvas y aletas, respectivamente. Se amplificaron cinco loci descritos por Barroso et al. (2003) para Brycon opalinus (número de Acceso GeneBank AF513621-BoM1, AF513622-BoM2, AF513623-BoM5, AF513626-BoM7 y AF513628-BoM13). Las reacciones se realizaron en un termociclador Eppendorf Mastercycler Gradient, programado para 30 ciclos, con un paso inicial de desnaturalización a $94^{\circ} \mathrm{C}$ por 4 min y un paso final de extensión a $72^{\circ} \mathrm{C}$ por $10 \mathrm{~min}$. Cada ciclo consistió de 1 $\min$ a $94^{\circ} \mathrm{C}, 1 \mathrm{~min}$ a $54^{\circ}, 60^{\circ}, 51^{\circ}, 51^{\circ}$ y $51^{\circ} \mathrm{C}$ para cada locus y $1 \min$ a $72^{\circ} \mathrm{C}$.

Las muestras amplificadas se sometieron a electroforesis en gel de poliacrilamida $10 \%$ (acrilamida:bisacrilamida, 29:1) desnaturalizado (6 mol L-1 de urea), conducidas en solución amortiguadora TBE $1 \mathrm{X}\left(90 \mathrm{mmol} \mathrm{L}^{-1}\right.$ Tris-Borato, $2 \mathrm{mmol} \mathrm{L}^{-1}$ EDTA) a $350 \mathrm{~V}(250 \mathrm{~mA})$, durante 6 horas. Para visualizar los alelos microsatélites, se utilizó la coloración con nitrato de plata descrita por Bassam et al. (1991), con algunas modificaciones. El gel se sometió a una solución de fijación (10\% etanol, 0,5\% ácido acético) por $20 \mathrm{~min}$, se tiñó con $6 \mathrm{mmol} \mathrm{L}^{-1} \mathrm{de}$ nitrato de plata por $30 \mathrm{~min}$ y, enseguida, previo lavado con agua destilada, se reveló con $0,75 \mathrm{~mol} \mathrm{~L}^{-1} \mathrm{NaOH}$ y $0,22 \%$ formol $40 \%$. Cada gel se fotografió con una cámara digital Cannon A520 4.0 Mp.

El tamaño de los alelos se calculó por el programa Kodak EDAS-290 (1D image analysis 3.5), utilizando DNA ladder (Invitrogen, EUA) de 100 pb. El tipo (A, $\mathrm{B}$ y C) y el número de pares de bases, observados en los reproductores y progenie de $B$. orbignyanus (141$200 \mathrm{pb}$ ) con los loci microsatélite, se organizaron en matrices de datos que se sometieron a los programas computacionales. El número de alelos, la heterozigosidad observada (Ho) y esperada (He), el índice de Shannon, la distancia e identidad genética y el número efectivo de alelos fueron calculados por el programa PopGene 1.31 (Yeh et al., 1999). La frecuencia alélica y la deficiencia o exceso de heterocigotos se calcularon para cada locus con el programa Genepop 1.2 (Raymond \& Rousset, 1995). El test del equilibrio de Hardy-Weinberg y el desequilibrio de ligación se calcularon con el programa Arlequin 3.1 (Excoffier et al., 2005), por el método de la cadena de Markov. Para analizar el desequilibrio de ligación, los grupos de parentales, larvas y alevinos, formaram tres agrupamientos: parentales x larvas, parentales $\mathrm{x}$ alevinos y larvas $\mathrm{x}$ alevinos. La diversidad genética de Nei (1973) y el coeficiente de endogamia $\left(\mathrm{F}_{\mathrm{is}}\right)$ se calcularon con el programa FSTAT 2.9.3.2 (Goudet, 2002).

\section{Resultados y Discusión}

Todos los reproductores usados en el cruzamiento sobrevivieron al proceso reproductivo, y todos los machos y hembras produjeron semen y óvulos. La utilización del sistema seminatural disminuyo significativamente la mortalidad de reproductores 
durante el proceso reproductivo. La preservación de los reproductores posibilita que un mayor número de individuos contribuya con la progenie y permite la preservación de la variabilidad genética. Por ese motivo, cuando el objetivo son programas de repoblación, el sistema reproductivo seminatural debe ser implementado (Povh, 2007).

Los cinco loci utilizados produjeron un total de 14 alelos, de los cuales fueron observados tres alelos (BoM1, BoM5, BoM7 y BoM13) y dos alelos (BoM2) por locus, presentes en los parentales y en la progenie (larvas y alevinos) (Cuadro 1). Se observaron tamaños de alelos entre 135 y $200 \mathrm{pb}$; el menor estuvo presente en el locus BoM5 (135 pb) y el mayor en el locus BoM2 (200 pb).

Em general, fueron observados alelos de alta frecuencia en los parentales y en la progenie, y no fueron encontrados alelos exclusivos. Sin embargo, fue observado un alelo raro (alelos con frecuencia menor que $0,05-5 \%$ ), en los alevinos, para el locus BoM7 (alelo $\mathrm{C}=0,009$ ) y alelos de baja frecuencia (menor que $0,100-10 \%$ ) en los parentales, para el locus BoM1 (alelo $\mathrm{C}=0,092)$, y en las larvas (BoM1, alelo $\mathrm{C}=0,060$ y BoM5, alelo $\mathrm{C}=0,087)$. El bajo número de alelos por locus ( 3 y 2 alelos) observado en este trabajo puede estar relacionado a la dificultad en la utilización de los loci heterólogos (desarrollados para Brycon opalinus), lo que hace sugerir la utilización de otros loci en la realización de estudios genéticos sobre $B$. orbignyanus. Estos resultados divergen de los encontrados por Barroso et al. (2003), quienes observaron 12 y 31 alelos (78 a $210 \mathrm{pb)} \mathrm{para} \mathrm{B.} \mathrm{opalinus.} \mathrm{Entretanto,} \mathrm{algunos}$ estudios han determinado la dificultad en la utilización de loci heterólogos en peces y otras especies. Lopera Barrero (2007) analizó la diversidad genética de lotes de $B$. orbignyanus y observó un bajo número de alelos (dos por locus), lo que indica la dificultad en la utilización de loci heterólogos. Igualmente, Sanches \&
Galetti Junior (2006), al amplificar loci microsatélite desarrollados para Brycon hilarii en B. orbignyanus, observaron un bajo polimorfismo (tres alelos para los loci Bh15 y Bh16). Barbosa et al. (2008), al verificar la amplificación de loci desarrollados para Prochilodus argenteus, en Prochilodus costatus y Prochilodus lineatus, verificaron amplificación monomórfica y la presencia de un bajo número de alelos en varios loci. Santos et al. (2009), al amplificar loci desarrollados para Colossoma macropomum en Pygocentrus nattereri, Mylossoma aureum y Piaractus brachypomus, observaron una baja amplificación de alelos (dos por locus) en cinco loci.

Las frecuencias de los alelos se mantuvieron estables en los parentales y en la progenie, con frecuencia desigual para cada locus. Para los loci BoM1, BoM2, BoM5 y BoM13, el alelo de mayor frecuencia fue el A, mientras que para el locus BoM7 el alelo B fue el que presentó mayor valor. En todos los loci, el número de alelos $(\hat{A})$ fue mayor que el número efectivo de alelos $\left(\hat{\mathrm{A}}_{\mathrm{e}}\right)$ (Cuadro 1), lo que confirma la distribución desigual de las frecuencias alélicas con la presencia de alelos de baja frecuencia, lo que puede influenciar la presencia de heterocigotos y provocar disminución de la variabilidad genética (Innes \& Elliott, 2006).

La presencia de los mismos alelos en los reproductores y en la progenie, la ausencia de alelos exclusivos y su frecuencia similar indican que hubo preservación de la variabilidad genética de los parentales en la progenie. Sin embargo, la presencia de pocos alelos en el lote (2 y 3 alelos por locus), los alelos de baja frecuencia, observados en los parentales y en las larvas, y el alelo raro, observado en el locus BoM7 de los alevinos, indican que la población de reproductores está en proceso de pérdida de alelos por disminución de la variabilidad genética (Bengtsson et al., 1995). Esto implica una alteración de la composición genética de la población de reproductores mantenidos en condiciones

Cuadro 1. Número de alelos por locus (Â), tamaño en pares de bases (pb), frecuencia de los alelos (A/B/C), número efectivo de alelos (Âe) y riqueza alélica (Ra), para los parentales y para la progenie de Brycon orbignyanus, en el sistema reproductivo seminatural.

\begin{tabular}{|c|c|c|c|c|c|c|c|c|c|c|c|}
\hline \multirow[t]{2}{*}{ Loci } & \multirow[t]{2}{*}{$\hat{\mathrm{A}}$} & \multirow[t]{2}{*}{$\mathrm{pb}$} & \multicolumn{3}{|c|}{ Parentales } & \multicolumn{3}{|c|}{ Larvas } & \multicolumn{3}{|c|}{ Alevinos } \\
\hline & & & Frecuencia & $\hat{\mathrm{Ae}}$ & $\mathrm{Ra}$ & Frecuencia & $\hat{\mathrm{Ae}}$ & $\mathrm{Ra}$ & Frecuencia & $\hat{\mathrm{Ae}}$ & $\mathrm{Ra}$ \\
\hline BoM1 & 3 & $168-144$ & $0,500 / 0,408 / 0,092$ & 2,354 & 3 & $0,509 / 0,431 / 0,060$ & 2,234 & 3 & $0,500 / 0,381 / 0,119$ & 2,443 & 3 \\
\hline BoM2 & 2 & $200-170$ & $0,488 / 0,512$ & 1,999 & 2 & $0,485 / 0,515$ & 1,998 & 2 & $0,478 / 0,522$ & 1,996 & 2 \\
\hline BoM5 & 3 & $143-135$ & $0,393 / 0,345 / 0,262$ & 2,923 & 3 & $0,529 / 0,384 / 0,087$ & 2,299 & 3 & $0,463 / 0,287 / 0,250$ & 2,783 & 3 \\
\hline BoM7 & 3 & $198-188$ & $0,192 / 0,423 / 0,385$ & 2,748 & 3 & $0,272 / 0,478 / 0,250$ & 2,739 & 3 & $0,107 / 0,884 / 0,009$ & 1,261 & 3 \\
\hline BoM13 & 3 & $172-160$ & $0,468 / 0,250 / 0,282$ & 2,708 & 3 & $0,488 / 0,226 / 0,286$ & 2,750 & 3 & $0,619 / 0,254 / 0,127$ & 2,112 & 3 \\
\hline
\end{tabular}


de cautiverio por seis años. Entretanto, de forma general, no ocurrió pérdida significativa de alelos en la formación de la progenie, ni hubo gran cantidad de alelos de baja frecuencia, lo que indica que el proceso de pérdida de alelos puede estar iniciando en la población de reproductores y que, según Wasko et al. (2004), es irreversible en lotes mantenidos en cautiverio.

La heterocigosidad observada (Ho) en los parentales fue alta para los loci BoM1, BoM5, BoM7 y BoM13 $(1,000)$, mientras que para el locus BoM2 fue menor $(0,537)$ (Cuadro 2). Al ser analizados por sexo, se observó igualmente una alta heterocigosidad observada y esperada $(\mathrm{He})$ (hembras: $\mathrm{Ho}=0,900$ y $\mathrm{He}=0,609$; machos: $\mathrm{Ho}=0,916$ y $\mathrm{He}=0,603)$. Estos resultados muestran que existe una adecuada variabilidad genética intra-poblacional, a pesar de que los reproductores estaban en cautiverio había seis años sin la introducción de nuevos individuos. Este hecho hace suponer que el plantel de reproductores se formó a partir de un número grande de reproductores que permitió conservar un adecuado perfil genético, y que no fue, posiblemente, influenciado por el efecto fundador, que es la pérdida

Cuadro 2. Heterocigosidad observada (Ho) y esperada $(\mathrm{He})$, coeficiente de endogamia $\left(\mathrm{F}_{\text {is }}\right)$ y test de probabilidad para el equilibrio de Hardy-Weinberg $\left(\mathrm{P}_{\mathrm{HW}}\right)$, estimados en los parentales, larvas y alevinos de Brycon orbignyanus, en el sistema reproductivo seminatural.

\begin{tabular}{llllc}
\hline Loci & Ho & He & $\mathrm{F}_{\text {is }}$ & $\mathrm{P}$ \\
\hline BoM1 & $1,000^{*}$ & 0,583 & $-0,733$ & $(0,0000)^{*}$ \\
BoM2 & $0,537^{\text {ns }}$ & 0,506 & $-0,062$ & $(0,0013)^{*}$ \\
BoM5 & $1,000^{*}$ & 0,666 & $-0,511$ & $(0,0000)^{*}$ \\
BoM7 & $1,000^{*}$ & 0,644 & $-0,563$ & $(0,0000)^{*}$ \\
BoM13 & $1,000^{*}$ & 0,638 & $-0,578$ & $(0,0000)^{*}$ \\
\hline Promedio & $0,907^{*}$ & 0,607 & $-0,489$ & - \\
\hline \multicolumn{5}{c}{ Larvas } \\
BoM1 & $0,898^{*}$ & 0,557 & $-0,651$ & $(0,0000)^{*}$ \\
BoM2 & $0,522^{\text {ns }}$ & 0,503 & $-0,098$ & $(0,0016)^{*}$ \\
BoM5 & $0,942^{*}$ & 0,569 & $-0,663$ & $(0,0000)^{*}$ \\
BoM7 & $0,956^{*}$ & 0,642 & $-0,498$ & $(0,0000)^{*}$ \\
BoM13 & $1,000^{*}$ & 0,641 & $-0,566$ & $(0,0000)^{*}$ \\
\hline Promedio & $0.864^{*}$ & 0,582 & $-0,496$ & - \\
BoM1 & $1,000^{*}$ & 0,595 & $-0,689$ & $(0,0000)^{*}$ \\
BoM2 & $0,377^{*}$ & 0,503 & $0,252^{(1)}$ & $(0,0007)^{*}$ \\
BoM5 & $0,926^{*}$ & 0,645 & $-0,440$ & $(0,0000)^{*}$ \\
BoM7 & $0,214^{\text {ns }}$ & 0,209 & $-0,026$ & $(0,0011)^{*}$ \\
BoM13 & $0,657^{*}$ & 0,530 & $-0,218$ & $(0,0020)^{*}$ \\
\hline Promedio & $0,635^{*}$ & 0,496 & $-0,224$ & - \\
\hline
\end{tabular}

${ }^{(1)}$ Perdida de heterocigotos. ${ }^{\text {ns No significativo. }{ }^{*} \text { Significativo a } 1 \% \text { de }}$ probabilidad. de variación genética cuando una población se forma a partir de un número pequeño de individuos proveniente de una población mayor (Caujapé-Castells, 2006). Esta afirmación es corroborada por los valores de índice de Shannon $(0,961)$ e de diversidad genética de Nei $(0,604)$, que ratifican una variabilidad genética intra-grupal, a pesar del proceso de pérdida de alelos relatado.

En la progenie, fueron encontrados valores de heterozigosis observada (Ho) entre $0,522-1,000$, para las larvas, y 0,214-1,000 para los alevinos (Cuadro 2), que caracterizan la presencia de una moderada variabilidad genética intra-poblacional, corroborada por los valores de heterocigosidad observada media $(0,864$ para larvas y 0,635 alevinos), índice de Shannon (0,918 para larvas y 0,798 para alevinos) y diversidad genética de Nei $(0,580$ para larvas y 0,497 para alevinos). Sin embargo los valores de heterocigosis observada (Ho) presentaron desvío de los valores de heterocigosidad esperada $(\mathrm{He})$ en los reproductores y en la progenie (larvas y alevinos), lo que caracteriza los desvíos significativos en el equilibrio de Hardy-Weinberg observados (Cuadro 2). Según Romana-Eguia et al. (2004) esto es siempre esperado en lotes mantenidos en cautiverio, ya que las desviaciones en las frecuencias, provocadas eventualmente por la deriva genética, tienden a ampliarse con el paso de las generaciones.

El desequilibrio de ligación, que es la asociación no aleatoria de alelos en dos o más loci (Caujapé-Castells, 2006), se observó en seis diferentes pares en los tres agrupamientos analizados: BoM1xBoM5, BoM1xBoM7, BoM1xBoM13, BoM5xBoM7, BoM5xBoM13, BoM7xBoM13. Los valores estimados del coeficiente de endogamia $\left(F_{\text {is }}\right)$ mostraron que en los parentales hubo exceso de heterocigotos para todos los loci, lo que caracterizó una adecuada variabilidad genética en el plantel de reproductores. Los valores estimados de $\mathrm{F}_{\text {is }}$ para las larvas mostraron, igualmente, exceso de heterocigotos (valores negativos de $F_{\text {is }}$ ). Entretanto, para los alevinos se observó déficit solamente en el locus BoM2 (Cuadro 2); esto confirma el proceso de endogamia evidenciado por los valores de heterocigosidad observada, índice de Shannon y diversidad genética de Nei.

El valor médio de Ho fue mayor en las larvas $(0,864)$ en comparación con los alevinos $(0,635)$, y menor si comparada con los parentales $(0,907)$; hubo una mayor identidad genética y una menor distancia genética entre los parentales y las larvas (Cuadro 3). 
Estos resultados muestran, al igual que el índice de Shannon y la diversidad genética de Nei, una tendencia a la disminución de la variabilidad genética durante las dos fases de crecimiento, con mayor intensidad en los alevinos, los que denota la presencia de un proceso de endogamia $\left(\mathrm{F}_{\text {is }}\right)$ en ese grupo.

La mayor semejanza de los parentales y las larvas y la menor variabilidad presente en los alevinos muestran la importancia de un manejo adecuado de los lotes y progenies, usados en los programas de repoblación de ríos. Según Lopera Barrero et al. (2008b), la primera providencia a ser tomada en la implantación de pisciculturas o de programas de repoblación es investigar la variabilidad genética de los lotes mantenidos en cautiverio. Una vez realizado ese análisis, es necesario el evaluar genéticamente las progenies que posteriormente serán liberadas en los ríos. La evaluación de las progenies va a depender directamente del tamaño de los alevinos a ser liberados, lo que es determinado por las condiciones ambientales definidas para cada lugar donde va a ocurrir la repoblación. Alevinos mayores son exigidos en ambientes con gran número de predadores, no obstante, lugares donde hay equilibrio de las relaciones presa/predador, alevinos menores y en mayor número pueden ser una estrategia adecuada (Sirol \& Britto, 2006).

Dependiente de cualquiera de esas dos condiciones de tamaño de liberación, el análisis genético de progenies es adaptado, y en la mayoría de las veces son tomadas muestras de larvas de tres a cinco días para analizar integralmente la variabilidad genética, presumiéndose que la muestra es representativa para los individuos que serán repoblados 60 o 90 días después. Los resultados encontrados en este estudio muestran que el valor de Ho de las larvas $(0,864)$ fue diferente del encontrado en los alevinos $(0,635)$ y que hubo pérdida de variabilidad genética en la progenie con mayor intensidad en los alevinos, lo que presumiblemente fue

Cuadro 3. Identidad genética (encima de la diagonal) y distancia genética (debajo de la diagonal), estimados para los parentales, larvas y alevinos de Brycon orbignyanus, en el sistema reproductivo seminatural.

\begin{tabular}{lccc}
\hline & Parentales & Larvas & Alevinos \\
\hline Parentales & - & 0,9798 & 0,8996 \\
Larvas & 0,0205 & - & 0,8379 \\
Alevinos & 0,1058 & 0,1769 & - \\
\hline
\end{tabular}

influenciado primeramente por la fase de canibalismo, presente normalmente en esta especie en la fase larval (Borba et al., 2006), por las condiciones ambientales de crecimiento, por la fase de aclimatación o por la mortalidad aleatoria. Sin embargo, no es posible hacer inferencias sobre la real influencia de estas hipótesis, ya que no se dispone de informaciones relacionadas y compiladas durante las diferentes fases de desarrollo.

De esta forma, en la evaluación genética de progenies destinadas a programas de repoblación, se hace evidente la necesidad de realizar el análisis en tiempos diferentes, dependiendo de la edad en que los individuos van a ser liberados en los ríos. Por los resultados obtenidos en este trabajo y sabiendo de la influencia ambiental y de competición que existe en las fases de crecimiento de los peces, es sugerido que en las progenies destinadas a programas de repoblación se realice primeramente un análisis genético en la fase larval ( 3 días), el cual va a ofrecer una visión genética general de la nueva generación. Enseguida, a los 60 o 90 días, a depender del manejo y de las condiciones ambientales del ecosistema donde van a ser liberados, realizar un segundo análisis para determinar objetivamente la real variabilidad genética con que los individuos van a ser liberados en el río y, así, determinar la verdadera viabilidad de la repoblación. Para los análisis genéticos se recomienda la utilización de otros loci heterólogos que permitan complementar y obtener un mayor polimorfismo genético que el obtenido en este trabajo o iniciar investigaciones que permitan desarrollar loci exclusivos para B. orbignyanus.

\section{Conclusiones}

1. La población de reproductores está en proceso de pérdida de alelos por disminución de la variabilidad genética.

2. Fie observada una mayor distancia genética entre los parentales y los alevinos, con pérdida de variabilidad genética.

3. Se verificó diferenciación genética entre larvas y alevinos.

\section{Agradecimientos}

A la Duke Energy International, por proveer los materiales para este trabajo; y a los estudiantes y técnicos que contribuyeron con la investigación. 


\section{Referencias}

AGOSTINHO,A.A.; THOMAZ, S.M.; GOMES, L.C. Conservation of the biodiversity of Brazil's inland waters. Conservation Biology, v.19, p.646-652, 2005.

BARBOSA, A.C.D.R.; GALZERANI, F.; CORRÊA, T.C.; GALETTI JUNIOR, P.M.; HATANAKA, T. Description of novel microsatellite loci in the Neotropical fish Prochilodus argenteus and cross-amplification in $P$. costatus and $P$. lineatus. Genetics and Molecular Biology, v.31, p.357-360, 2008.

BARROSO, R.M.; HILSDORF, A.W.S.; MOREIRA, H.L.M.; MELLO, A.M.; GUIMARÃES, S.E.F.; CABELLO, P.H.; TRAUB-CSEKO, Y.M. Identification and characterization of microsatellites loci in Brycon opalinus (Cuvier, 1819) (Characiforme, Characidae, Bryconiae). Molecular Ecology Notes, v.3, p.297-298, 2003.

BASSAM, B.J.; CAETANO-ANOLLÉS, G.; GRESSHOFF, P.M. Fast and sensitive silver staining of DNA in polyacrylamide gels. Analytical Biochemistry, v.196, p.80-83, 1991.

BENGTSSON, B.O.; WEIBULL, P.; GHATNEKAR, L. The loss of alleles by sampling: a study of the common outbreeding grass Festuca ovina over three geographic scales. Hereditas, v.122, p.221-238, 1995.

BORBA, M.R.; FRACALOSSI, D.M.; PEZZATO, L.E. Dietary energy requirement of piracanjuba fingerlings, Brycon orbignyanus, and relative utilization of dietary carbohydrate and lipid. Aquaculture Nutrition, v.12, p.183-191, 2006.

CAUJAPÉ-CASTELLS, J. Brújula para botánicos desorientados en la genética de poblaciones. Las Palmas de Gran Canaria: Exegen, 2006. 133p.

EXCOFFIER, L.; LAVAL, G.; SCHNEIDER, S. Arlequin version 3.1: an integrated software package for population genetics data analysis. Evolutionary Bioinformatics Online, v.1, p.47-50, 2005.

GOUDET, J. FSTAT: a program to estimate and test gene diversities and fixation indices (version 2.9.3.2). Lausanne: University of Lausanne, Department of Ecology \& Evolution, 2002. Disponível em: <http://www2.unil.ch/popgen/softwares/fstat.htm>. Acesso em: 21 dez. 2009.

INNES, B.H.; ELLIOTT, N.G. Genetic diversity in a Tasmanian hatchery population of Atlantic salmon (Salmo salar L.) compared with its Canadian progenitor population. Aquaculture Research, v.37, p.563-569, 2006.

LOPERA-BARRERO, N.M. Diversidade genética de Brycon orbignyanus em sistema reprodutivo seminatural. 2007. 92p. Tese (Doutorado) - Universidade Estadual de Maringá, Maringá.

LOPERA-BARRERO, N.M.; POVH, J.A.; RIBEIRO, R.P.; GOMES, P.C.; JACOMETO, C.B.; LOPES, T. da S. Comparación de protocolos de extracción de ADN con muestras de aleta y larva de peces: extracción modificada con cloruro de sodio. Ciência e Investigación Agraria, v.35, p.77-86, 2008a.

LOPERA-BARRERO, N.M.; RIBEIRO, R.P.; POVH, J.A. $\mathrm{O}$ repovoamento de peixes: uma estratégia multidisciplinar? Aqüicultura \& Pesca, v.30, p.71-74, 2007.
LOPERA-BARRERO, N.M.; RIBEIRO, R.P.; POVH，J.A.; GOMES, P.C.; VARGAS, L.; OLIVEIRA, S.N. Caracterización genética de lotes de peces usados en programas de repoblamiento y su importancia en la conservación genética en la piscicultura. Zootecnia Tropical, v.26, p.515-522, 2008b.

LOPERA-BARRERO, N.M.; RIBEIRO, R.P.; SIROL, R.N.;POVH, J.A.; GOMES, P.C.; STREIT JUNIOR, D.P.; VARGAS, L.M. Caracterización genética de lotes de Brycon orbignyanus utilizados en programas de repoblamiento. Revista MVZ Córdoba, v.13, p.1110-1119, 2008c.

MACHADO, A.B.M.; MARTINS, C.S.; DRUMMOND, G.M. (Ed.). Lista da fauna brasileira ameaçada de extinção: incluindo a lista das espécies quase ameaçadas e deficientes em dados. Belo Horizonte: Fundação Biodiversitas, 2005. 157p.

NEI, M. Analysis of gene diversity in subdivided populations. Proceedings of the National Academy of Sciences of the United States of America, v.70, p.3321-3323, 1973.

PEDREIRA, M.M. Comparação entre três sistemas no cultivo de larvas de piracanjuba (Brycon orbignyanus). Revista Ceres, v.50, p.779-786, 2003.

POVH, J.A. Avaliação da diversidade genética e do manejo reprodutivo do pacu, Piaractus mesopotamicus. 2007. 75p. Tese (Doutorado) - Universidade Estadual de Maringá, Maringá.

POVH, J.A.; LOPERA-BARRERO, N.M.; RIBEIRO, R.P.; LUPCHINSKI JUNIOR, E.; GOMES, P.C.; LOPES, T.S. Monitoreo genético en programas de repoblamiento de peces mediante marcadores moleculares. Ciencia e Investigación Agraria, v.35, p.5-15, 2008.

POVH, J.A., RIBEIRO, R.P., SIROL, R.N., STREIT JUNIOR, D.P. Diversidade genética de pacu do Rio Paranapanema e do estoque de um programa de repovoamento. Pesquisa Agropecuária Brasileira, v.43, p.201-206, 2008.

RAYMOND, M.; ROUSSET, F. An exact test for population differentiation. Evolution, v.49, p.1280-1283, 1995.

ROMANA-EGUIA, M.R.R.; IKEDA, M.; BASIAO, Z.U.; TANIGUCHI, N. Genetic diversity in farmed Asian Nile and red hybrid tilapia stocks evaluated from microsatellite and mitochondrial DNA analysis. Aquaculture, v.236, p.131-150, 2004.

SANCHES, A.; GALETTI JUNIOR, P.M. Microsatellites loci isolated in the freshwater fish Brycon hilarii. Molecular Ecology Notes, v.6, p.1045-1046, 2006.

SANTOS, M. da C.F.; HRBEK, T.; FARIAS, I.P. Microsatellite markers for the tambaqui (Colossoma macropomum, Serrasalmidae, Characiformes), an economically important keystone species of the Amazon River floodplain. Molecular Ecology Resources, v.9, p.874-876, 2009.

SIROL, R.N.; BRITTO, S.G. Conservação e manejo da ictiofauna: repovoamento. In: NOGUEIRA, M.G.; HENRY, R.; JORCIN, A. (Ed.). Ecologia de reservatórios: impactos potenciais, ações de manejo e sistemas em cascatas. São Carlos: RiMA, 2006. p.275-284.

TAYLOR, B.W.; FLECKER, A.S.; HALL JUNIOR, R.O. Loss of a harvested fish species disrupts carbon flow in a diverse tropical river. Science, v.313, p.833-836, 2006. 
WASKO, A.P.; MARTINS, C.; OLIVEIRA, C.; SENHORINI, J.A.; FORESTI, F. Genetic monitoring of the Amazonian fish matrinchã (Brycon cephalus) using RAPD markers: insights into supportive breeding and conservation programmes. Journal of Applied Ichthyology, v.20, p.48-52, 2004.

YEH, F.C.; BOYLE, T.Y.Z.; XIYAN, J.M. PopGene Version 1.31:

Microsoft Window-based freeware for population genetic analysis.
Alberta: University of Alberta; Center for International Forestry Research, 1999. 29p.

ZANIBONI FILHO, E.; REYNALTE-TATAJE, D.; WEINGARTNER, M. Potencialidad del género Brycon en la piscicultura brasileña. Revista Colombiana de Ciencias Pecuarias, v.19, p.233-240, 2006.

Recibido el 27 abril de 2009 y aceptado el 9 diciembre de 2009 